\title{
Primary Fallopian Tube Carcinoma: Results of a Single-Institutional Retrospective Analysis of 17 Patients with Evaluation of Staging and Prognostic Factors
}

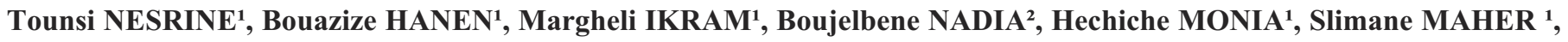 \\ Rahel KHALED ${ }^{1}$
}

Tunis, Tunisia

\section{ABSTRACT}

OBJECTIVE: Primary carcinoma of the fallopian tubes is one of the less common gynecological cancers. It constitutes $(0.14-0.18 \%)$ of gynecological malignancies. Our study aimed to review the managing process of primary carcinoma of the fallopian tubes in the mono-center institute and to identify prognostic factors impacting survival.

STUDY DESIGN: A retrospective cohort study regarding patients with fallopian tube carcinoma treated between July 1991 and December 2005 was identified at the Tunisian anticancer institute "Salah Azaiez". During this period, we have identified 17 patients. Data such as age, gravidity and parity, menopausal condition, symptoms reported by the patient on presentation, adjuvant therapy, stage of illness, surgical intervention, pathological findings, tumor recurrence, and previous surgical procedures were obtained from the patients' reports.

RESULTS: The average age at the time of diagnosis was 58 years. Fourteen of the included patients were postmenopausal. Surgery was the initial therapy for 15 patients. Optimal cytoreductive surgery was achievable in seven patients with no residual tumors. Histologic examination revealed that serous adenocarcinoma type was the predominant type. Two were in stage I and, four were in stage II; seven were in stage III and four in stage IV. The median follow-up time was 24 months. At the time of the final analysis, 11 patients died of disease. 5-year OS, DFS was $21 \%$ and $37 \%$ respectively. In our study, only the residual tumor was a significant prognostic factor predicting survival.

CONCLUSION: Complete optimal surgery with no residual tumor was the main goal of the surgeon to improved survival in primary fallopian tube carcinoma.

Keywords: Cytoreductive surgery, Primary carcinoma of the fallopian tubes, Prognostic factor, Residual tumor

Gynecol Obstet Reprod Med 2021;27(3):279-282

${ }^{1}$ Department of Surgical Oncology Salah-Azaiez Institute, Boulevard of 9 Avril, 1001 Tunis, Tunisia

${ }^{2}$ Department of Pathology, Salah-Azaiez Institute, Boulevard of 9-Avril, 1001 Tunis, Tunisia

Address of Correspondence: Tounsi Nesrine

Department of Surgical Oncology, SalahAzaiez Institute, Boulevard of 9-Avril, 1001

Tunis, Tunisia

neserine.tounsi@gmail.com

Submitted for Publication: 20.02.2020 Revised for Publication: 24.05.2020 Accepted for Publication: 24.03.2021 Online Published: 31.03.2021

ORCID IDs of the authors: TN: 0000-0002-8845-4773

$H B$ : 0000-0001-5885-1252, IM: 0000-0001-5830-5040,

BN: 0000-0002-4888-8198, HM: 0000-0002-7165-447X

SM :0000-0003-0311-847X, KR:0000-0002-3474-0651

\begin{tabular}{cc}
\hline Quick Response Code: & Access this article online \\
\cline { 2 - 2 } & Website: www.gorm.com.tr \\
& e- mail: info@gorm.com.tr \\
\cline { 2 - 2 } & DOI:10.21613/GORM.2021.1075 \\
\hline
\end{tabular}

How to cite this article: Nesrine T. Hanen B. Ikram M. Nadia B. Monia H. Maher S. Khaled R. Primary Fallopian Tube Carcinoma: Results of a SingleInstitutional Retrospective Analysis of 17 Patients with Evaluation of Staging and Prognostic Factors. Gynecol Obstet Reprod Med. 2021;27(3):279-282

\section{Introduction}

Primary carcinoma of the fallopian tubes (PFTC) is uncommon gynecological cancer. It constitutes $(0.14-0.18 \%)$ of gynecological malignancies $(1,2)$.PFTC had several points in common as ovarian cancer, especially factor's risk (hormonal, reproductive, and genetic factors) $(3,4)$. Also, the management of PFTC shares similar concerns and principles as ovarian carcinoma (4). However, because of late diagnostic and early lymphogenic metastases, PFTC had a poor prognosis (5).

Our study aimed to review the managing process of PFTC in the mono-center institute and to identify prognostic factors impacting survival.

\section{Material and Method}

A retrospective cohort study regarding patients with fallopian tube carcinoma treated between July 1991 and 
December 2005 was identified at the Tunisian anticancer institute "Salah Azaiez". During this period; we have identified 17 patients. After having had the agreement of our ethics committee for using data. Data such as age, gravidity and parity, menopausal condition, symptoms reported by the patient on presentation, adjuvant therapy, stage of illness, surgical intervention, pathological findings, tumor recurrence, and previous surgical procedures were obtained from the patients' reports. Consent for using data were obtained. All procedures were performed according to the Declaration of Helsinki.

Relative to operation information and a result of the pathology, patients were classified according to the staging system of the International Federation of Gynecology and Obstetrics (FIGO)-2014.

Univariate analyses of possible prognostic factors (age, FIGO stage, histological type, tumor grade, and residual tumor) were performed.

Kaplan Meier life table analysis was used to generate survival curves. Survival based on categorical variables was compared using the log-rank test. Data were analyzed using SPSS (Statistical Package for the Social Sciences) software version 20 (IBM Corp. Armonk, NY, USA). A p-value less than 0.05 was considered significant.

\section{Results}

The average age at the time of diagnosis was 58 years \pm 8 (range, 43-75 years). Fourteen of the included patients were postmenopausal. A history of infertility was found in two patients.

The mean parity was $4.9 \pm 3$ with a range of 0 to 10 . No patient has used hormone replacement therapy. Four patients had used oral contraceptive pills.

Two patients had personnel medical history of carcinoma, one patient had breast cancer declared 6 years ago; the other was treated for rectal adenocarcinoma declared 5 years ago. No history of family cancer was reported.

The presenting symptoms were: a palpable pelvic and/or abdominal mass in seven cases, followed by abnormal vaginal bleeding in six cases, discharge abdominal pain in 8 patients, lymphatic metastasis inguinal or supraclavicular in 3 patients, Pleurisy in one case.

Two patients had a preoperative diagnosis of endometrial cancer, three had surgical procedures due to benign reasons (hydrosalpinx, menometrorrhagia, postmenopausal bleeding).

For the remaining patients, ovarian cancer was the most common diagnostic discussed.

Cancer antigen 125 (CA-125) was measured preoperatively in 11 patients and was found to be elevated in eight cases with a median of $200 \mathrm{U} / \mathrm{mL}$.
Surgery was the first therapy for 15 patients. Only two patients with stage IV had received a neoadjuvant-chemotherapy with a good response.

Optimal cytoreductive surgery was achievable in seven patients with no residual tumors. Seven patients had gone through pelvic and para-aortic lymphadenectomy patients. The mean number of resected lymph nodes (LN) was ten. Positive lymph node metastases were observed in 4/7 patients.

From patients who have had a residual tumor, four patients had a postoperative residual tumor with a diameter greater than $1 \mathrm{~cm}$.

Histologic examination revealed that serous adenocarcinoma type was histologically predominant in 9 patients. Three patients had a low level, three had a high grade and six had a moderately differentiated grade. The demographic and morphological characteristics of the patients are summarized in table I.

Table I: Demographic and morphological features

\begin{tabular}{|c|c|}
\hline Parameter & No \\
\hline \multicolumn{2}{|l|}{ Age (years) } \\
\hline$\leq 60$ & 2 \\
\hline$>60$ & 15 \\
\hline Menopause & 14 \\
\hline \multicolumn{2}{|l|}{ FIGO Stage } \\
\hline 1 & 2 \\
\hline II & 4 \\
\hline III & 7 \\
\hline IV & 4 \\
\hline \multicolumn{2}{|l|}{ Surgical procedures: } \\
\hline$>\mathrm{TAH}+\mathrm{BS} 0+\mathrm{TO}+$ cytology & 10 \\
\hline > $\mathrm{TAH}+\mathrm{BSO}+\mathrm{RPLND}+\mathrm{TO}+$ cytology+appendectomy & 4 \\
\hline $\begin{array}{l}>\mathrm{TAH}+\mathrm{BSO}+\mathrm{RPLND}+\mathrm{TO}+\text { cytology+ RS-RA+ } \\
\text { appendectomy }\end{array}$ & 3 \\
\hline \multicolumn{2}{|l|}{ Residual tumor at initial surgery $(\mathrm{cm})$} \\
\hline$\leq 1$ & 8 \\
\hline$>1$ & 9 \\
\hline \multicolumn{2}{|l|}{ Chemotherapy } \\
\hline platinum salts and cyclophosphamide & $5 / 14$ \\
\hline$>$ paclitaxel and carboplatin & $10 / 14$ \\
\hline
\end{tabular}

TAH: Total abdominal hysterectomy, BSO: Bilateral salpingooophorectomy, RPLND: Retroperitoneal pelvic lymph node dissection (pelvic + para-aortic), TO: Total omentectomy, RS-RA: Rectosigmoid resection and anastomosis

Two were in stage I and, four were in stage II, seven were in stage III, and four in stage IV.

Fourteen patients underwent adjuvant chemotherapy protocol consisting of 6-8 cures of paclitaxel and carboplatin for 10 patients. For the remaining patients, they had platinum salts and cyclophosphamide according to the protocol established at that time. No patient received radiotherapy.

The median follow-up time was 24 months (range 4-193 
months). Six patients had no recurrence in the follow-up period, six did have disease recurrence and five had progressive disease.

At the time of the final analysis, 11 patients died of disease. 5-year OS, DFS was $21 \%$ and $37 \%$ respectively.

In our study, only the residual tumor was a significant prognostic factor predicting survival $(p=0.001)$.

However; FIGO stage, histology differentiation, and age were not significant survival prognostic factors of PFTC (Table II).

Table II: Predicted factors for survival in univariate analysis

\begin{tabular}{lccc}
\hline Factor & $\mathbf{n}$ & Median (OS) & $p$ (log-rank) \\
\hline Age (years) & & & \\
$<50$ & 2 & 7 & 0.289 \\
$>50$ & 15 & 37 & \\
FIGO & 6 & 55 & 0.09 \\
I, II & 11 & 20 & \\
III & & & \\
Residual tumor & 8 & 55 & 0.001 \\
No & 9 & 19 & \\
Yes & 9 & & 0.8 \\
Histology & 9 & 24 & \\
Serous & & & \\
\hline
\end{tabular}

\section{Discussion}

Primary carcinoma of the fallopian tubes is a rare tumor(6). It shares several points with epithelial ovarian cancer (EOC) such as histological and epidemiological features.

Recent data suggest that the true incidence of primary fallopian tube carcinoma has been substantially underestimated, this conclusion is based on compelling evidence that papillary serous carcinoma, the most common subtype of epithelial ovarian carcinoma, actually arises from the epithelial lining of the fallopian tube $(7,8)$.

Due to the rarity of this cancer, there are very limited data concerning prognostic factors influencing OS and response to chemotherapy.

It is difficult to distinguish PFTC from EOC based on clinical manifestations. Some authors had demonstrated that MRI could be used to differentiate between primary fallopian tube carcinoma and epithelial ovarian cancer(9).

However, if we base on histological analyses to distinguish between primary ovarian and primary tubal cancer, many characteristics should be evaluated: Tumor volume, distribution of tumor in the ovaries (diffuse or partial), and the presence of intraepithelial carcinoma (for tubal and endometrial origins). Partial involvement of ovaries was associated with larger tumors in the peritoneum, and these were considered to be peritoneal in origin. Diffuse involvement of the ovaries was also suggestive of an ovarian origin. The presence of intraepithelial serous carcinoma in the fallopian tubes was indicative of a tubal origin(10).

In our study, most of the patients had an advanced stage. Only two patients had neoadjuvant chemotherapy. For the remaining patients, surgery was the first step of treatment. Residual tumor was left for nine patients.

5-year OS and DFS were $21 \%$ and $37 \%$ respectively. Only the residual tumor was a significant prognostic factor predicting survival $(p=0.001)$.

Our data is in accordance with other studies, the overall 5year survival was ranging from a low of $22 \%$ to a high of $57 \%$ for all stages of PFTC $(3,5)$.

Some authors advocated many prognostic factors associated with survival. The most one was the FIGO stage $(3,4,11,12)$. Other prognostic factors were advocated in the literature: include patient age at the time of diagnosis, residual tumor after initial surgery, and histologic tumor grade $(4,12)$.

Even though our present study failed to demonstrate that the FIGO stage was an independent prognostic factor for survival, because of the lesser number of patients with earlystage (two cases). Most of the patients in our series had advanced stage; this might also help to explain the worse survival rate in this present study.

In the study of Akkaya, et al (3), multiple factors were found to influence the survival probability: FIGO stage, the pathological type of the tumor, the diameter of the residual tumor after surgery, the tumor grade, preoperative CA-125 levels, and the presence of ascites.

Nevertheless, in the study of Lau et al, age, tumor, stage, histology type, and grading were not significantly related to OS (13).

The poor prognosis of PFTC is also due to the richest lymphatic networks that supplied fallopian tubes.

Therefore, PFTC has a propensity for early lymphatic spread and a higher rate of retroperitoneal and distant metastases relative to EOC (14).

Seidman, et al have reported that it was a correlation between lymphatic invasion in the fallopian tubes and the frequency of distant metastases (15).

Lymph node metastases have been reported in 33\% of cases with different PFTC stages (16). Nonetheless, PFTC rarely gives rise to metastasis in supraclavicular lymph nodes (17). In our study, one patient had supraclavicular lymph nodes metastases, and two patients presented to our institute with inguinal lymph nodes metastases as first presented symptoms. 
Finally, our study has some limitations. It is a retrospective study and a small number limits us. Most of the patients had reached an advanced stage and this could limit the statistical power. Moreover, our study supports that optimal surgery with no residual tumor was the main goal of the surgeon to improved survival.

Further studies are needed to better understand the risk factors of PFTC, and a multicenter randomized, clinical trial is necessary to determine the most favorable medical management protocols to be applied.

Acknowledgments: We want to acknowledge the archive department of the Salah Aziez Institute of oncology in Tunis for their help.

Availability of data and materials:The data supporting this study is available through the corresponding author upon reasonable request.

Conflict of Interest: The authors do not report any financial or personal connections with other persons or organizations, which might negatively affect the content of this publication and/or claim authorship rights to this publication.

Financial Support: None

Authors' contributions: NT:, HB:, and IM: Raised the presented idea. NT:, HB: and NB: Designed the study. NB: Conducted the analyses. NT: and HB: Developed the first draft of the manuscript. MH: Participated in data analysis, interpretation, and draft revision. MS: and MH: Participated in data collection and result interpretation. IM:, NT:, and KR: Assisted with data collection and analysis. MS: Designed the study and critically revised the manuscript. All authors read and approved the final manuscript.

\section{References}

1. Nanaiah SP, Rathod PS, Rajkumar NN, Kundargi R, Subbian A, Ramachandra PV, et al. Primary carcinoma of the fallopian tube: a review of a single institution experience of 8 cases. Scientific World Journal. 2014;2014: 630731. Doi:10.1155/2014/630731.

2. Pectasides D, Pectasides E, Economopoulos T. Fallopian tube carcinoma: a review. Oncologist. 2006;11(8):902-12. Doi:10.1634/theoncologist.11-8-902.

3. Akkaya E, Sanci M, Kulhan NG, Kulhan M, Nayki U, Nayki C, et al. Prognostic factors of primary fallopian tube carcinoma. Contemp Oncol (Pozn). 2018;22(2):99104. Doi:10.5114/wo.2017.69590.

4. Rosen AC, Ausch C, Hafner E, Klein M, Lahousen M, Graf AH, et al. A 15-year overview of management and prognosis in primary fallopian tube carcinoma. Austrian Cooperative Study Group for Fallopian Tube Carcinoma. Eur J Cancer. 1998;34(11):1725-9. Doi:10.1016/s09598049(98)00214-7.

5. Kalampokas E, Kalampokas T, Tourountous I. Primary fallopian tube carcinoma. Eur J Obstet Gynecol Reprod Biol.
2013;169(2):155-61. Doi:10.1016/j.ejogrb. 2013. 03.023.

6. Rosenblatt KA, Weiss NS, Schwartz SM. Incidence of malignant fallopian tube tumors. Gynecol Oncol. 1989;35 (2):236-9. Doi:10.1016/0090-8258(89)90051-6.

7. Sun M, Bao L, Shen H, Ji M, Yao L, Yi X, et al. Unexpected primary fallopian tube carcinoma during gynecological operations: Clinicopathological and prognostic factors analyses of 67 cases. Taiwan J Obstet Gynecol. 2019;58(5):626-32. Doi:10.1016/j.tjog.2019.07.008.

8. Gungorduk K, Ertas IE, Ozdemir A, Akkaya E, Telli E, Taskin S, et al. Prognostic significance of retroperitoneal lymphadenectomy, preoperative neutrophil lymphocyte ratio and platelet lymphocyte ratio in primary fallopian tube carcinoma: A multicenter study. Cancer Res Treat. 2015;47(3):480-8. Doi:10.4143/crt.2014.058.

9. Yang Y, Xiao Z, Liu Z, Lv F. MRI can be used to differentiate between primary fallopian tube carcinoma and epithelial ovarian cancer. Clin Radiol. 2020;75(6):457-465. Doi:10.1016/j.crad.2020.02.002.

10. Diniz PM, Carvalho JP, Baracat EC, Carvalho FM. Fallopian tube origin of supposed ovarian high-grade serous carcinomas. Clinics (Sao Paulo). 2011;66(1):73-6. Doi:10.1590/s1807-59322011000100013.

11. Schneider C, Wight E, Perucchini D, Haller U, Fink D. Primary carcinoma of the fallopian tube. A report of 19 cases with literature review. Eur J Gynecol Oncol. 2000; 21(6):578-82. PMID: 11214613.

12. Gadducci A, Landoni F, Sartori E, Maggino T, Zola P, Gabriele A, et al. Analysis of treatment failures and survival of patients with fallopian tube carcinoma: a cooperation task force (CTF) study. Gynecol Oncol. 2001;81 (2):150-9. Doi: 10.1006/gyno.2001.6134.

13. Lau HY, Chen YJ, Yen MS, Chen RF, Yeh SO, Twu NF. Primary fallopian tube carcinoma: a clinicopathologic analysis and literature review. J Chin Med Assoc. 2013; 76(10):583-7. Doi: 10.1016/j.jcma.2013.06.010.

14. Hiensch R, Meinhof K, Leytin A, Hagopian G, Szemraj E, Epelbaum O. Clinically occult primary fallopian tube carcinoma presenting as a malignant pleural effusion. Clin Respir J. 2017;11(6):1086-90. Doi:10.1111/crj.12423.

15. Seidman JD, Krishnan J. Lymphatic invasion in the fallopian tube is a late event in the progression of pelvic serous carcinoma and correlates with distant metastasis. Int J Gynecol Pathol. 2020;39(2):178-83. Doi:10.1097/ PGP.0000000000000580.

16. Tamimi HK, Figge DC. Adenocarcinoma of the uterine tube: potential for lymph node metastases. Am J Obstet Gynecol. 1981; 141(2):132-7. Doi:10.1016/s0002-9378 (16)32579-0

17. Eken M, Temizkan O, Kaygusuz EI, Herkiloğlu D, Çöğendez E, Karateke A. Primary carcinoma of the fallopian tubes: Analysis of sixteen patients. Turk J Obstet Gynecol. 2015;12(2):83-8. Doi:10.4274/tjod.67355. 